\title{
La belleza del código. El desarrollo de HTML5
}

\section{The beauty of the code. The rising of HTML5}

\section{Raúl Tabarés Gutiérrez}

Fundación TECNALIA RESEARCH \& INNOVATION

Faraondemetal@gmail.com

Fecha de aceptación definitiva: 09/03/2015

\section{Resumen}

Gracias al crecimiento, desarrollo y popularización de la World Wide Web, su desarrollo tecnológico goza de un mayor protagonismo en la sociedad, debido a la creciente integración de ambas. Nuestro ánimo en este artículo es describir, caracterizar y analizar el proceso de creación y desarrollo del nuevo estándar web de hipertexto; HTML5. Al mismo tiempo, exploramos este proceso a la luz de varias teorías que aúnan tecnología y sociedad.

\section{Abstract}

Thanks to the growth, development and popularization of World Wide Web, its technological development has a greater importance in society due to the growing embedding of both of them. Our aim in this article is to describe, to characterize and to analyze the process of rising and development of the new web hypertext standard; HTML5. At the same time we explore this process in light of various theories about technology and society. We also pay particular attention to World Wide Web users and the general 
También prestamos especial atención a los usuarios de la World Wide Web y al uso que éstos hacen de los «Social Media» o «Medios Sociales». Sugerimos que los usuarios, a través de estas aplicaciones, ejercen una influencia en el desarrollo tecnológico de la Web y de los estándares en particular.

Palabras Clave: World Wide Web; Web; Internet; Tim Berners-Lee; HTML; HTML5; CSS3; JavaScript; SCOT; tecnología autónoma; innovación; innovación de usuario; innovación abierta; historia social media; usuario; navegador web; WhatWG; API; difusión social; innovación social. usage of "Social Media». We suggest that users influence web standards particularly, and technological development too through these kinds of new technologies.

\section{Introducción}

Actualmente, la creación y desarrollo de estándares web está afrontando un punto de inflexión debido a la mayor presencia e influencia que tienen en la sociedad, tanto los dispositivos móviles como los medios sociales.

Si bien es cierto que la World Wide Web es una tecnología que a su vez se basa en tres tecnologías, que son; El protocolo HTTP (Hypertext Transfer Protocol), el localizador URI (Unified Reference Indentificator) y el lenguaje de marcado HTML (Hypertext Markup Language). Constatamos que solamente esta última ha afrontado recientemente, grandes cambios en su desarrollo tecnológico y para el cual, puede entreverse un buen número de interrelaciones sociales en su conceptualización y desarrollo. Por lo tanto, creemos que su estudio desde un punto de vista que auné tecnología y sociedad, puede ser de sumo interés.

Este artículo describe el origen y desarrollo de HTML5 (Hypertext Markup Language), enuncia sus características más disruptivas y analiza desde un punto de vista social cuales han sido sus principales hitos. Nuestro ánimo en este artículo es contrastar teorías que explican la dinámica social de la 
tecnología (como el modelo SCOT), con otras que enuncian su pérdida de control por parte de la sociedad (como la Tecnología Autónoma) y al mismo tiempo evidenciar el papel cada vez más importante que juegan los usuarios en el desarrollo tecnológico y de estándares. Sugerimos que los usuarios son una de las fuentes de la innovación tecnológica y ejercen una gran influencia a la hora de desarrollar estándares.

\section{El desarrollo de HTML5}

\subsection{La creación de HTML5}

El nacimiento de HTML5 constituye un punto de inflexión grande, en el modo que se desarrollan los estándares web. El origen de HTML5 tiene su origen en una "pequeña revolución» de profesionales de compañías privadas, disconformes con el rumbo que había decidió tomar la organización que vela por el desarrollo de los estándares, el W3C. Un grupo de desarrolladores de la Fundación Mozilla y de Opera Software, a los que se les unen más tarde profesionales de Apple, fundan en 2004 el Web Hypertext Application Technology Working Group (WhatWG), al margen del W3C (Franganillo 2010).

Este hecho sucede en junio de 2004, cuando el W3C alberga un seminario sobre «Web Applications and Compound Documents»1. En esta conferencia se dieron cita distribuidores de navegadores web, compañías de desarrollo web y otro tipo de agentes que integraban el W3C en esos momentos. Durante este evento, profesionales de Opera y Mozilla realizan una presentación sobre la visión que tenían en aquellos momentos acerca del futuro de la web y qué se podía resumir en una premisa; "Evolucionar el HTML4 existente en un estándar capaz de incluir nuevas características para los desarrolladores de aplicaciones web» (Pilgrim 2010)

Además, subrayaron la importancia de su propuesta en torno a 7 principios (The Mozzilla Foundation \& Opera Software 2004):

1. Se puede consultar los detalles de este «workshop» en el siguiente enlace: http://www. w3.org/2004/04/webapps-cdf-ws/ (Accedido por última vez el 23/12/2013) 
- Backwards Compatibility, clear migration path

Las tecnologías web que se utilicen, deben ser tecnologías, con las que los desarrolladores se encuentren familiarizados, como HTML, CSS, DOM y JavaScript. Por otro lado, las características a implementar y las nuevas funcionalidades a soportar, deben ser en base a las que soporta el navegador Internet Explorer 6, ya que es el que mayor cuota de mercado tiene, y por lo tanto, a lo que la mayoría de los usuarios están acostumbrados.

- Well-defined error handling

El manejo de los errores en las aplicaciones web debe ser definido a un detalle en el cual los desarrolladores no tengan que inventárselos o utilicen procesos de reingeniería basándose en cómo los maneja la competencia.

- Users should not be exposed to authoring errors

Las especificaciones deben especificar exactamente como se deben recuperar los errors, para cada escenario posible.

- Practical use

Cada nueva aplicación web que se genere, debe ser justificada por un uso práctico. (Esto no tiene la misma validez en el caso contrario).

- Scripting is here to stay

Pero también debe evitarse, cuando pueda ser sustituido por el «lenguaje de marcas".

- Device-specific profiling should be avoided

Los desarrolladores deben ser capaces de implementar las mismas funcionalidades de la página, ya sea accedida a la versión de escritorio o a través de un dispositivo móvil.

- Open process

La web se ha beneficiado de su desarrollo en un entorno abierto. Las aplicaciones web, serán de carácter primordial para la web y por lo tanto, su desarrollo deberá ser realizado de una manera abierta (listas de correo, drafts, etc).

Aparte de esta presentación y propuesta, también sondearon la opinión de los asistentes mediante una encuesta, en la cual lan Hickson, de Opera Software, preguntaba: «Should the W3C develop declarative extension to HTML and CSS and imperative extensions to DOM, to address medium level 
Web Application requirements, as opposed to sophisticated, fully-fledged OS-level APIs?»

La votación fue de 11 votos en contra y 8 a favor. Además de esta negativa, el W3C hizo una declaración formal en la que indicaba que no iba a prestar apoyo ni ofrecer recursos a ninguna propuesta de desarrollo que no estuviera dentro de los grupos de trabajo que tenía establecidos en esa época (PILGRIM 2010). Debido a esta negativa del W3C, el grupo de personas que habían hecho esta propuesta decidieron continuar su camino fuera del paraguas del W3C. Por ello, registraron el dominio whatwg.org y comenzaron a trabajar en su propuesta, fundando el WHAT Working Group (Web Hypertext Applications Technology Working Group)2. Este grupo de trabajo no oficial y abierto a la colaboración de terceros, se caracterizaba por estar formado por fabricantes de navegadores y agentes interesados que querían volver a los orígenes de HTML y no ahondar en lenguajes nuevos (como XHT$\mathrm{ML}$ ). Su enfoque se basaba en garantizar la compatibilidad regresiva o retrocompatibilidad $^{3}$, del nuevo estándar. Una característica muy apreciada entre los usuarios y desarrolladores de lenguajes de programación y que sin duda contribuye a la coherencia de las trayectorias del desarrollo tecnológico.

Sin embargo, el W3C quería proseguir con el desarrollo de XHTML, el cual no tiene compatibilidad regresiva con HTML. Necesita de un «MIME type» nue$v^{4}{ }^{4}$ y suponía tirar por la borda todo el trabajo realizado anteriormente con HTML. Los navegadores siempre se «han olvidado» de los errores sintácticos de HTML y nadie se había preocupado hasta entonces, por como especificarlos. La historia del desarrollo web hasta este momento, se había basado en buena parte, en como los diferentes desarrolladores de navegadores hacían compatibles sus productos, con los de los competidores, ignorando las especificaciones y estándares. La mayoría de los navegadores se centraban en «poder presentar», de la mejor manera posible, «las sopas de etiquetas» que se creaban (Andersson 2007). El WHAT Working Group dedicó 5 años de trabajo para documentar adecuadamente como analizar y diseccionar

2. Se puede consultar la web que alojó este grupo de «insurrectos» y su declaración de intenciones en este enlace; http://www.whatwg.org/news/start

3. Traducido del inglés; "Backwards Compatibility»

4. Los MIME Types (Multipurpose Internet Mail Extensions) constituyen la especificación del tipo de documento que se intercambia. Se señala en el comienzo del código de las páginas web. Se puede ampliar la información respecto al concepto en sí en la página en castellano de la Wikipedia. http://es.wikipedia.org/wiki/Multipurpose_Internet_Mail_Extensions 
HTML, de un modo compatible con las páginas existentes 5 (Pilgrim 2010). Aparte de esta ingente tarea, este grupo también trabajó en el soporte nativo de audio y video (sin plugins), la nueva etiqueta "canvas» (que permite «dibujar» directamente sobre la estructura del documento) y otra especificación en torno a aplicaciones web. El motivo de la fundación de este grupo es la disconformidad respecto a la visión que tenía de los estándares el W3C. Para el WhatWG, el enfoque que hacía el W3C era demasiado académico y por ello, la creación de este grupo aspiraba a la creación de una nueva versión del estándar, pero desde un punto de vista eminentemente práctico. El W3C sin embargo parecía estar buscando en diferentes tecnologías un sustituto a HTML y especialmente se fijaba en XHTML 2.0 (O'Mara 2012).

Lo cierto es que después de unos dos años y medio después de esa conferencia y los dos caminos separados que tomaron el W3C y el WAHTWG, estaba claro que XHTML 2 languidecía mientras las nuevas características de HTML levantaban grandes expectativas. Este hecho, se unía a las voces críticas que acusaban al W3C de un avance lento y pocos resultados concretos (Castro 2007). Por eso, en octubre de 2006, Tim Berners-Lee y el W3C anunciaron que trabajarían junto al WAHTWG para añadir nuevas funcionalidades y evolucionar de manera conjunta XHTML. (Berners-Lee 2006)

Posteriormente, en octubre del 2009, el W3C cerraría el grupo de trabajo en torno a XHTML2 (Le Hegaret 2009) y pararía completamente sus actividades en este lenguaje, para volcarse de lleno en el desarrollo de HTML5, del cual se publicaría en 2008 un «working draft»(Hickson and Hyatt 2008), gracias a la unión de esfuerzos de las 2 organizaciones. En los años siguientes, los navegadores comienzan a soportar HTML5 (Mozilla Firefox es el primero en dar el paso) y se empieza una fase de divulgación, difusión y concienciación en torno a esta tecnología. Sin embargo, el mayor impacto (desde el punto de vista de difusión social), se produce cuando Steve Jobs publica "Thoughts on Flash", haciéndose eco del mayor peso de los dispositivos móviles a la hora de desarrollar estándares y del problema que genera software propietario como «Flash». Steve elogiaba a HTML5, CSS3 y Javascript por su funcionalidad y por el hecho de constituir estándares abiertos (Jobs 2010). Otras compañías y plataformas con gran poder de prescripción social como Youtube (Harding 2010), Slideshare (Slideshare-Blog 2011) y Scribd

5. Este documento de referencia sigue actualizándose y puede consultarse en el siguiente enlace http://www.whatwg.org/specs/web-apps/current-work/multipage/parsing.html 
(Calore 2010) también mostraron su apoyo a HTML5 y su intención de implementar esta tecnología en sus aplicaciones, en la medida en que satisficiera sus necesidades de mercado.

Desde entonces y hasta ahora, el desarrollo de HTML5 ha seguido de manera imparable su curso. Actualmente HTML5 es una «W3C Candidate Recommendation", (W3C 2011) con la vista puesta en 2014, para alcanzar un estándar definitivo.

\subsection{Las mejoras de HTML5}

Lo primero que debemos especificar en torno a HTML5, es que no es una tecnología, sino un conjunto de tecnologías. Esto es importante, ya que hasta ahora, junto al protocolo HTTP (Hypertext Transfer Protocol) y al identificador URI (Universal Reference Identificator), HTML formaba un trío de tecnologías clave para la World Wide Web. A partir de ahora, el paradigma de HTML5 engloba una serie de tecnologías que se recogen bajo un mismo estándar, pero que multiplican en gran medida las posibilidades del desarrollo web. Y es que dentro de este estándar, van a tener cabida multitud de elementos dinámicos y multimedia, en forma de "etiquetas", que reconfiguran el entorno web y los contenidos, tal y como los conocíamos. La especificación de HTML5 no se compone de una sintaxis únicamente, sino que admite dos sintaxis; HTML y XHTML (Extensible HyperText Markup Language). Así, los desarrolladores web pueden elegir entre un enfoque práctico, pero poco riguroso (HTML) o un enfoque académico y estricto (XHTML). El W3C ha aceptado que HTML y XHTML sean recomendaciones paralelas que puedan coexistir (Franganillo 2010). HTML5 no está basado en SGML (Standard Generalized Markup Language) y está diseñado para que tenga compatibilidad regresiva tanto con HTML4 como con XHTML1 (Chau 2009). Además de estructurar los documentos, HTML5 también aporta una gran mejora, que no es otra que la de cómo se deben interpretar los errores y poner fin de paso a la "guerra entre navegadores» que existía, para cómo se deben subsanar los errores. (Andersson 2007; Keith 2010)

HTML5 presenta nuevos elementos (o etiquetas) destinados a ordenar y enriquecer la presentación de los documentos. Son muestra de ello elementos de carácter semántico como article, header, hrgroup, nav, section, 
aside y footer. Los blogs y web's de noticias han influido en gran medida en la conceptualización de estos nuevos elementos (Schafer 2010), ya que han introducido dinamismo en las web's y sus contenidos y la necesidad de una cronología, para poder organizar la información. En particular, el elemento article, es uno de los que el W3C ha introducido, para recoger más información sobre la página y está diseñado en base a los posts de un blog (W3C 2013).

En cuanto a la recogida de información, el medio típico a través del cual un usuario envía datos a un servidor es a través de los formularios. Éstos recogen la información que introducen los usuarios y la envían a aplicaciones que se ejecutan en el servidor. HTML5 incorpora un gran número de controles como email, range, date, time, placeholder, autofocus, etc, que ejercen su función sin necesidad de utilizar Javascript. Un lenguaje de programación que se ejecuta a través de un software que no tienen porqué tener instalado los usuarios de la web (Pilgrim 2010). Gracias a estas mejoras, HTML5 hace posible que sean los navegadores quienes faciliten la entrada y validación de datos, que tienen un patrón regular o están sometidos a restricciones. (Franganillo 2010) Este laborioso trabajo, ahora se descarga sobre los navegadores, en vez de sobre los diseñadores.

Aparte de estos elementos de estructura del lenguaje, los aspectos más innovadores de la sintaxis de HTML5 son sin duda, sus elementos dinámicos y multimedia. Muchos de ellos han sido creados tras agrias polémicas y la liberación de «códecs» en formato propietario (Linder 2013; Schonfeld 2010). Hay que especificar también que aunque HTML5 no sea soportado por los viejos navegadores, esto no tiene importancia, ya que HTML5 no es técnicamente un lenguaje nuevo, sino una colección de nuevos elementos que pueden ser soportados por los nuevos navegadores (Pilgrim 2010). HTML5 propone una nueva forma de estructurar la información de las páginas web, pero manteniendo la compatibilidad regresiva (tan importante en el desarrollo de estándares). Pero no sólo eso, ya que además provee de nuevas API's ${ }^{6}$ (Application Programation Interface), para reforzar la experiencia de usuario, pero sin la introducción de elementos procedentes de software propietario, sino fomentando la estandarización. Algunos de los elementos más reseña-

6. API, del inglés «Application Programation Interface», es una Interfaz de Programación de Aplicaciones que permite interactuar a un software con otro, gracias a un conjunto de funciones y procedimientos que se ofrecen a través de una biblioteca de recursos. 
bles e innovadores de esta nueva versión de HTML son las etiquetas audio, video, canvas o geolocation, entre otras muchos. Además de los elementos dinámicos que introduce HTML5 en su sintaxis, también hay otros dos lenguajes que complementan el espectro de este conjunto de innovaciones. Por ello, no se puede entender HTML5 sin CSS3 (Cascading Style Sheets); ya que es el lenguaje que regula los aspectos de estilo de las páginas web; y tampoco sin Javascript; ya que este último permite programar acciones dinámicas y facilitar la interacción con el usuario. Por último, también se ha lanzado una nueva versión del DOM (Document Object Model), que consiste en la colección de objetos que representan los elementos del lenguaje HTML en la página web.

\section{La creación de HTML5 desde un punto de vista social}

El proceso que da origen al desarrollo de HTML5 es un caso de estudio en sí mismo por la idiosincrasia del mismo. Muchas veces se ha incidido en el hecho de que la industria de los distribuidores de navegadores no han respetado las normas en cuanto a los procesos de estandarización y han promovido innovaciones que han generado muchos problemas, de cara a la normalización de los lenguajes implicados ${ }^{7}$. Pero en este caso, el «boicot» que lideran los integrantes de compañías como Opera, Mozilla y Apple, se debe a que precisamente es el W3C (que es la organización que vela por el desarrollo de los estándares), la que no decide apostar por una nueva versión de HTML. Por ello, las personas de estas compañías involucradas lideran el movimiento WhatWG, para apostar por una nueva versión de HTML que incorpore elementos dinámicos y las API's tan necesarias a su vez para crear las nuevos tipos de página web y aplicaciones que habían sido popularizados al albor del fenómeno conocido como «Web 2.0».

7. Uno de los episodios más memorables que nos deja la historia de la Web, es el conocido como «La Guerra de Navegadores». En él, las dos compañías que pujaban por el dominio del mercado de los navegadores web (Microsoft y Netscape Communications), se enzarzaron en una carrera tecnológica por ofrecer el máximo de funcionalidades y características al usuario final. Este proceso conllevo la implementación de muchas funcionalidades en ambos navegadores sin el proceso de contraste y aprobación necesario para la estandarización de las mismas. Un buen ejemplo de esto es la extinta etiqueta «Marquee». Se puede ampliar la información al respecto en la página que la Wikipedia en inglés dedica a esta cuestión http://en.wikipedia.org/ wiki/Browser_wars 
Si bien hasta ahora hemos observado con detenimiento el desarrollo de HTML5 desde un punto de vista histórico y descriptivo. En este apartado, el objetivo es analizar, el proceso anteriormente descrito desde un punto de vista social y comprobar cómo los usuarios han jugado un papel determinante en el desarrollo del nuevo estándar. Para ello se han establecido tres epígrafes en los cuales se abordan tres enfoques relacionados con la sistemática del proceso innovador que se ha estudiado previamente y en el cual se ponen de relieve las diferentes conceptualizaciones sociales de la tecnología implicada. El ánimo aquí, es cubrir la mayor parte de variantes de lo que se entiende como un sistema técnico y sus diferentes grupos de usuarios implicados en su conceptualización, desarrollo, mantenimiento y re-conceptualización.

\subsection{Difusión e innovación social en HTML5}

La difusión social de las innovaciones es un elemento clave en su popularización y adopción social. Uno de los primeros autores en investigar el fenómeno de la difusión social de la innovación fue el sociólogo rural británico Everett Rogers, el cual publicó en 1962 el libro titulado; «Diffusion of Innovations», en el cual definía el proceso de difusión social como;

"Difusión es el proceso mediante el cual una innovación es comunicada a miembros de un sistema social a través de ciertos canales a lo largo del tiempo» (Rogers 1962)

El caso de HTML5, no ha sido diferente a esta lógica, ya que se han hecho múltiples esfuerzos en varios canales, con el fin de divulgar las ventajas de este nuevo paradigma innovador. La difusión social de HTML5 ha sido un proceso en el cual han estado involucrados tanto instituciones como el W3C, compañías privadas como Apple y Google, fundaciones sin ánimo de lucro como Mozilla y personalidades de la talla de Tim Berners-Lee (Berners-Lee 2006). Todos ellos han maniobrado en una misma dirección, con el fin de que el cambio de paradigma innovador que suponía HTML5, llegase a buen puerto. Si esta innovación no hubiese sido aceptada y adoptada, es de suponer que no hubiera tenido éxito (Echeverría 2013), ya que una innovación sin difusión, no tiene ningún impacto económico (OECD 2005) y por lo tanto 
no se populariza. Así mismo, una percepción pública negativa, también puede ser una barrera infranqueable para esa innovación (López Cerezo and González 2013). Por ello, una vez que el W3C accedió a colaborar en el desarrollo de HTML5, ha hecho mucho hincapié en la difusión de este nuevo paradigma innovador. Prueba de ello, son las campañas de marketing desarrolladas por el W3C, enfocadas en la difusión del estándar y que han contado con el desarrollo de logos específicos de HTML5, CSS3 y JavaScript (Figura 1). Quizás, una de las figuras públicas que más apoyo han prestado en este sentido, ha sido el malogrado Steve Jobs, con sus declaraciones a favor del nuevo estándar y en contra del software propietario como Flash (Jobs 2010). En una decisión bastante polémica, llevó incluso este apoyo hasta las últimas consecuencias, denegando el soporte a Flash, en su dispositivos móviles como iPhone, iPad e iPod.

\section{Figura 1. \\ Logotipos de HTML5, JavaScript y cSS3}

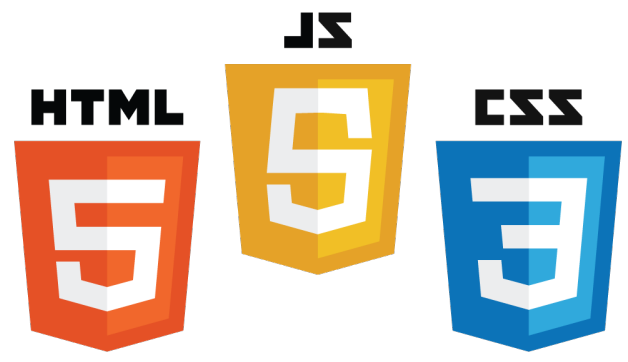

Por otro lado, también creemos que el proceso de creación de HTML5 constituye una innovación social en tanto que se cuestiona el "status quo" en cuanto a lo que estándares se refiere, se pone en entredicho al W3C y se promueve un proceso participativo para apoyar el desarrollo de una nueva versión de HTML de acuerdo a unos principios y valores abiertos y comunes. Una innovación social es relevante en la medida que se oriente a valores sociales (Echeverría 2008) y el proceso por el cual se genera HTML5 responde a 7 principios que salvaguardan tanto esos valores sociales, como una coherencia con el desarrollo tecnológico desarrollado hasta la fecha. Creemos que el proceso de creación de HTML5 no se limita a un proceso de desarrollo 
tecnológico al uso, sino que hay una compilación de saberes explícitos e implícitos que se mezclan con las innovaciones técnicas (Innerarity and Gurrutxaga 2009) que se desarrollan en este periodo de tiempo. La creación de HTML5 no pasa por la identificación de un problema nuevo, sino por la redefinición de un problema existente, al cual no se había enfrentado desde un marco conceptual adecuado, tal y como hemos expuesto anteriormente. Este sentido de innovación social que queremos recoger aquí, se aleja un poco del que promulga Geoff Mulgan (Mulgan et al. 2007; Mulgan 2006), en el sentido de que las organizaciones que promueven este cambio no tienen fines sociales ${ }^{8}$. No es nuestro objetivo aquí fijarnos en el tipo de agente que promueven estas innovaciones, sino reconocer y analizar el proceso que se genera. Por ello, creemos que la creación de HTML 5 abre la puerta a un nuevo paradigma de la innovación y es un movimiento que se genera desde abajo hacia arriba, volviendo a los propios valores que generaron la Web. Además, HTML5 asienta las bases para crear nuevas innovaciones en torno a unos principios sociales y tecnológicos que deben ser consensuados y respetados, para garantizar la cohesión y el desarrollo tecnológico sostenible.

\section{2. ¿Construcción social o tecnología autónoma?}

Al revisar el proceso de conceptualización y desarrollo de HTML5, llama poderosamente también la atención, la cantidad de actores implicados en su desarrollo, el número de lenguajes de programación, marcado y diseño (y por lo tanto, itinerarios tecnológicos a tomar) y las diversas interacciones que se producen entre estos agentes y las posibles trayectorias tecnológicas. Por ello, a la hora de recoger los fragmentos que la historia tecnológica de este desarrollo nos ha dejado por el camino, no es fácil elegir una metodología o un marco de actuación que nos empodere totalmente de cara a realizar una «foto» lo más precisa posible de todos estos hechos. Este es un tema que se ha repetido a lo largo de la historia de los Estudios Sociales de Ciencia y Tecnología, y en el cual ha habido varias escuelas y corrientes que han intentado dar su propia explicación de la dinámica del cambio tecnológico

8. Apple y Opera son compañías privadas. Por otro lado, Mozilla Foundation es una conocida fundación sin ánimo de lucro que tiene como misión «mantener la elección y la innovación en Internet». 
(Hackett et al. 2007). Durante muchas décadas, los estudios del desarrollo tecnológico han interpretado la tecnología en un sentido mucho más amplio que el que puede referirse a un artefacto o una herramienta en particular. Esta búsqueda por encontrar una definición los más completa posible ha generado una gran cantidad de literatura al respecto y ha expandido nuestra percepción utilitarista y artefactual de la tecnología (González García, López Cerezo y Luján 1996). Entre otros autores que han promovido una comprensión más holística de lo que significa el concepto de técnica y tecnología, nos gustaría mencionar a Jacques Ellul (Ellul 1964) y Miguel Ángel Quintanilla (M. Á. Quintanilla 1989). Estos dos autores han introducido en sus dos definiciones de técnica, el concepto de eficiencia, el cual se convierte en uno de los resortes de la dinámica social.

Los autores anteriormente mencionados, nos sirven de introducción al modelo SCOT ( Social Construction of Technology).El cual también es conocido como "constructivismo social» y que contempla a los usuarios de la tecnología como un grupo social que juega un papel fundamental en el desarrollo de las tecnologías involucradas (Bijker, Hughes, and Pinch 1987). Este modelo desarrollado por Bjiker, Hughes y Pinch, supuso un punto de inflexión en los estudios STS (Science, Technology and Society), a la hora de explicar la dinámica y caracterización del desarrollo tecnológico. En este enfoque, la idea de «sistema tecnológico» juega un papel muy importante, a la hora de comprender la dimensión social de la tecnología (Hughes 1987). La idea de "sistema tecnológico» es bastante similar a la que Quintanilla denomina como "sistema técnico», la cual describe como:

Un dispositivo complejo compuesto de entidades físicas y de agentes humanos, cuya función es transformar algún tipo de cosas para obtener determinados resultados característicos del sistema. (M. A. Quintanilla 1998)

Bijker también ahonda en el concepto de tecnología y establece a su vez, tres capas diferentes de significado:

Artefactos físicos, actividades humanas y conocimiento. (Bijker 1995)

En estas concepciones de técnica, tecnología, sistema técnico y sistema tecnológico, residen no sólo artefactos, sino también sujetos sociales, sistemas sociales y diversos tipos de conocimientos. Queremos hacer hincapié en este "sentido social» que tiene el desarrollo de la tecnología, ya que el 
caso que nos ocupa del desarrollo del nuevo estándar HTML5, creemos que encaja perfectamente bajo este marco teórico. El desarrollo del estándar no se lleva a cabo por un modelo de desarrollo tecnológico o de «innovación lineal» (Lundvall 1992), sino que es fruto de un proceso iterativo en el cuál hay una retroalimentación de diversos actores y paradigmas tecnológicos diversos. La construcción de este nuevo estándar creemos que es un proceso social, en el cual hay varios agentes implicados en su creación y desarrollo, en mayor o menor medida. Como ya hemos señalado previamente, el W3C es la organización que gestiona el desarrollo de estándares para la web, pero debido al estancamiento de sus propuestas en torno a XHTML y su difícil encaje, fueron perdiendo fuelle, a favor de la nueva propuesta que hicieron las personas implicadas de las organizaciones Opera Software, The Mozilla Foundation y Apple. En el modelo scot se enfatiza que la tecnología no determina el curso de la acción humana, sino más bien al contrario. La forma final de un artefacto tecnológico es el resultado de un proceso social, en el que diversos grupos sociales juegan un papel crucial en la selección de las variantes a elegir (Bijker and Pinch 1984), posibilitando la supervivencia de unas trayectorias y la extinción de otras. Este proceso es al que Bijker y Pinch enuncian como la «flexibilidad interpretativa» de la tecnología (Bijker, Hughes, and Pinch 1987; Bijker and Pinch 1984). Diferentes grupos de usuarios pueden construir diferentes significados para con la tecnología, pero a medida que el paradigma dominante se abre paso, los demás significados se desvanecen. Por ello, entender el papel que juegan estos grupos de usuarios es clave. En el caso que nos ocupa, el grupo de usuarios que formaron los desarrolladores de Firefox, Opera y Apple, es decisivo en el establecimiento del paradigma dominante en el desarrollo de HTML5 y de los valores que hay detrás de este nuevo paradigma tecnológico.

Este último punto nos lleva irremediablemente a tener que hablar de la relación de las tecnologías y los valores, un tema que toma forma expresa en un famoso artículo de Langdon Winner, titulado «Do artifacts have politics?» (Winner 1980). También, es el propio Winner quien realiza una influente crítica del modelo SCOT, por lo descriptivo y analítico del mismo y por lo poco crítico del modelo, respecto al desarrollo tecnológico (Winner 1993). Por ello, a pesar de que el modelo scot es de gran ayuda para describir la dinámica del desarrollo tecnológico (como el propio Winner admite) y explicar cómo es construida socialmente la tecnología, debemos también hacer mención al concepto de «Tecnología Autónoma» (Winner 1979), ya que la historia de 
HTML5 es una reacción en cierto modo a la situación «fuera de control» que se produce en el desarrollo de estándares debido al fenómeno «Web 2.0».

Tal y como define Winner, la idea de tecnología autónoma; "sirve de etiqueta a todas las concepciones y observaciones en el sentido de que la tecnología escapa de algún modo al control humano» (Winner 1979: 25). Como argumenta Winner, ciertas técnicas son capaces de desencadenar efectos no previstos o deseados. Esto es a lo que él se refiere como una peculiaridad de la tecnología del siglo XX, en el sentido de

La pérdida del dominio (sobre la tecnología) se manifiesta en una mengua en nuestra habilidad para conocer, juzgar o controlar nuestros medios técnicos. (Winner 1979: 38)

Lo cierto es que la corta, pero intensa historia de la web está llena de episodios en los cuales, el desarrollo de estándares es siempre uno de los mayores perjudicados. Esta pérdida del dominio tecnológico viene representada por varios factores que se dan en el desarrollo de la web, por motivos muy diversos, intereses encontrados y agentes dispares. Ejemplo de esta pérdida del dominio tecnológico es la proliferación de software propietario como Flash, a lo largo del periodo conocido como «Web 2.0» y que constituyen una gran amenaza a la interoperabilidad de la plataforma que en su día ideó Tim Berners-Lee. Otros episodios anteriores a este periodo de popularización de la web, como la famosa «Guerra de Navegadores» también ha dejado anécdotas cuando menos inquietantes como la proliferación de elementos extraños en el desarrollo de estándares como la etiqueta MARQUEE$^{9}$ (una etiqueta que hacía que el texto «bailara por la pantalla»). Este tipo de desarrollos, mejoras e innovaciones son una de las principales armas que utilizan los distribuidores de software y navegadores web, para presionar e ir «más allá de los estándares». La justificación de este énfasis por la mejora continua de las aplicaciones, es debido a que si dichas nuevas

9. Las etiquetas Marquee (IE) y Blink (Netscape) son dos ejemplos de cómo a veces el empuje de las compañías por innovar y ofrecer algo diferente al usuario puede convertirse en algo problemático que eche por tierra el trabajo ya realizado. Estas etiquetas se caracterizan por ofrecer textos que continuamente se mueven en la pantalla. Lo cual suponía un auténtico quebradero de cabeza para los usuarios que querían consultar información, sin constantemente tener distracciones en la pantalla. Además, también presentaban bastantes problemas en la optimización del código. Se puede consultar en la página de la Wikipedia en inglés esta información. http://en.wikipedia.org/wiki/Marquee_element [Consultado el 25/09/2012] 
funcionalidades son aceptadas y valoradas por una mayoría de usuarios de la web, suelen traducirse en una mayor cuota de mercado. Por ello, la mayoría de compañías que basan su modelo de negocio en la web presionan en esta dirección. El papel del W3C siempre ha sido el de velar por el que todos estos "empujes», no atenten contra la naturaleza de la Web e Internet y controlar la incorporación de elementos externos o propietarios al estándar.

\subsection{El poder de los usuarios}

Por último, en esta visión social del fenómeno HTML5 nos gustaría prestar atención a un último grupo, cuyo papel puede haber sido determinante en todo este proceso; los usuarios. Si bien hemos constatado como a través de una serie de agentes se promueve un cambio a favor de la re-estandarización de la web y el abandono de elementos externos o de software propietario, creemos que una de las mayores fuerzas de cambio que posee la World Wide Web son sus propios usuarios. Durante el fenómeno conocido como Web 2.0, el cual abarca un periodo de tiempo en torno a finales de lo '90 hasta finales de la siguiente década (es difícil cuantificar cuando se acaba esta época, ya que es un concepto difuso y en permanente evolución), se produce un gran incremento de usuarios de la web, debido en su mayor parte a la popularización de la misma, a través de la aparición de aplicaciones colaborativas (y que posteriormente se catalogarían como medios sociales).

Esta popularización de la web a través de este tipo de aplicaciones es uno de sus mayores puntos de inflexión, ya que contribuye en gran medida a su popularización. El creador de la Web, Tim Berners-Lee ha mostrado y manifestado en varias ocasiones su contrariedad a que la «primera versión» de la web no tuviera esa "centralidad» en las personas (Laningham 2006). Pero lo cierto es que en sus primeras etapas, la web es percibida como un medio en el cual solo podían publicar contenidos un reducido número de personas ${ }^{10}$, con los conocimientos necesarios (P. Anderson 2007) y es

10. Quizás, el origen del problema pueda estar en el propio desarrollo tecnológico inicial de la Web y en los primeros navegadores que se desarrollan; ViolawWw, Mosaic y Netscape, los cuales no traen integrada por defecto, una función de «editar» (Netscape en sus últimas versiones sí que implementaría de forma opcional un editor de HTML). 
innegable que hasta el desarrollo de la Web 2.0 no se percibe ésta como un producto de masas (Duggan and Smith 2014).

\section{Figura 2.}

Horas de vídeo subidas a YouTube desde junio de 2007 a mayo de 2013

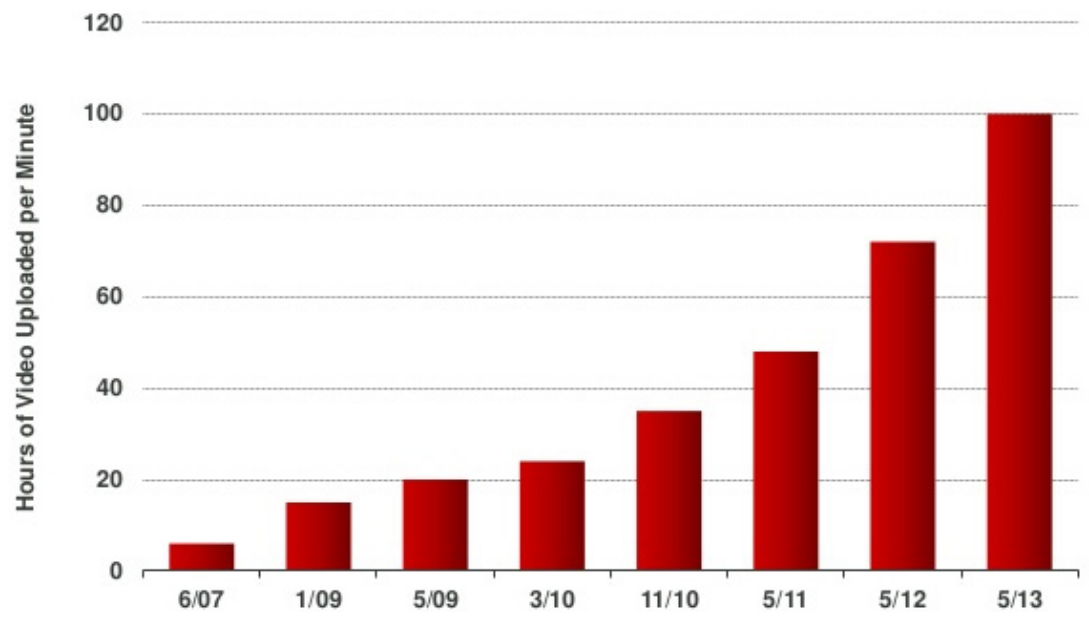

Fuente: KPCB a través de YouTube.

Además de atraer usuarios, la Web 2.0 también produce un gran cambio, en el tipo de contenidos que se producen en la misma, ya que a partir de este momento el multimedia (fotos, videos, etc.) pasa a cobrar un mayor protagonismo. Los usuarios que no disponen de los conocimientos necesarios a nivel de programación (HTML, CSS, JavasScript, PHP, etc.), ven en plataformas como Facebook, Blogger, YouTube o Flickr, la herramienta ideal que se adapta a sus necesidades y con la cual pueden compartir información e interactuar con otros usuarios. Las «tecnologías sociales» (Helmer, Brown, and Gordon 1966) que aparecen en este periodo constituyen la «caja de herramientas» que necesitaba el usuario no técnico para poder generar contenido e interactuar con otros usuarios, intercambiando información. Este tipo de procesos capacitadores, de cara a los usuarios, es lo que algunos autores han identificado como una "democratización de la innovación» (Von Hippel 2005). 
En nuestra opinión, estos primeros usuarios de este tipo de aplicaciones o «lead users» (Von Hippel 1998) son los principales causantes de la popularización de la web. La generalización de este tipo de servicios, de cara a crear y compartir contenidos, unidos a otras fuerzas como el «efecto red» (Klemperer 2006, Liebowitz and Margolis 1994; Shapiro and Varian 1999), «la economía de la larga cola» (C. Anderson 2004; C. Anderson 2007), el fomento de una «arquitectura de participación» (O’Reilly 2005) e incluso la consolidación en la Web de «tecnologías fáticas» (Wang, Tucker, and Rihll 2011; Tucker, Wang, and Haines 2012) producen el ecosistema idóneo para que los usuarios no técnicos ${ }^{11}$, puedan crear y compartir fácilmente información en la World Wide Web. A partir de la eclosión del fenómeno conocido como «Web 2.0" se rebajan las barreras de entradas de uso de las aplicaciones, promoviendo la sencillez en su uso, entornos más ligeros (que no consuman mucho ancho de banda) y que demuestren su interoperabilidad a través de API's (muchas veces explotando datos de los servicios, con el objetivo de que terceros puedan desarrollar «mash-up's» ${ }^{12}$ [P. Anderson 2007]).

\section{Figura 3.}

\section{Crecimiento de usuarios de Internet desde 1996}

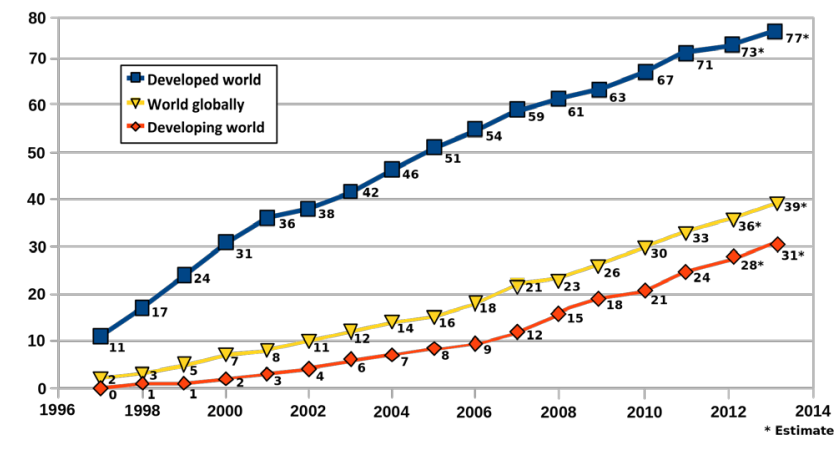

Fuente: Wikipedia.

11. Entendemos como usuario no técnico, aquel que no dispone de conocimientos de programación web. Es decir, ausencia de conocimientos de lenguajes como HTML, CSS, JavaScript, PHP, Pearl, Python, etc.

12. Un «Mash-Up» es una aplicación web, que se sirve de otras fuentes (a través de una API) para crear un servicio de agregación o de valor añadido. El sitio web http://digg.com/ es un ejemplo de «Mash-Up». 
Este nuevo ecosistema, hace posible que los usuarios también puedan editar la Web (aunque no directamente) y de este modo empezar a innovar en ella. El «Social Media» O «Medios Sociales» se convierte en la herramienta del usuario para poder crear y compartir contenidos, pero además, también es el elemento con el cual se influye en el desarrollo tecnológico. Esto es debido a que una mayor predominancia de elementos multimedia, hacen necesarios un mayor conjunto de elementos nativos de estandarización, ya que si se abusa de software propietario, se ralentiza la experiencia de usuario y la interoperabilidad entre servicios. También podemos decir, que al fin y al cabo, son los clásicos criterios de eficiencia de un sistema técnico, los que residen en esta argumentación (Ellul 1964; M. Á. Quintanilla 1989). Otros autores han definido este tipo de influencia también como "cultura», en el sentido de que sobrepasa las preferencias individuales e influye en el desarrollo tecnológico (Castells 2001)

Por otro lado, el peso de los usuarios también se nota directamente en el desarrollo puramente técnico del estándar, ya que como el propio W3C describe, el elemento article está diseñado en base a los posts de un blog (W3C 2013) y los propios blogs han influido en gran medida en la conceptualización de nuevos elementos (Schafer 2010) presentes en HTML5.

\section{Conclusiones}

En el presente artículo hemos explorado el proceso de creación y desarrollo de HTML5 tanto desde la perspectiva tecnológica, como desde la perspectiva social. El objetivo ha sido siempre el de proporcionar visiones complementarias, con el fin de diseccionar las interrelaciones que se producen entre estas dos perspectivas.

Tal y como hemos presentado, en el desarrollo de HTML5 existen elementos característicos del modelo scot y el "Constructivismo Social», pero al mismo tiempo, algunos de los efectos no deseados de la "Tecnología Autónoma». En contraposición a esta dualidad, también hemos explorado el papel de los usuarios en el proceso de creación del nuevo estándar y especialmente su influencia oculta, en la conceptualización del nuevo estándar y del paradigma de innovación que rige el desarrollo de los estándares web. 
En nuestro trabajo, sugerimos que los usuarios no técnicos han influido decisivamente, a través de la adopción y uso de medios sociales o «Social Media» en

a) La popularización y apropiación social de la World Wide Web.

b) La vuelta a la estandarización y el abandono de software propietario, a favor de HTML5.

c) La primacía de contenidos multimedia en la World Wide Web.

Como hemos descrito anteriormente, si bien esta influencia no se produce de manera directa (ya que los usuarios no editan directamente la web) y la cantidad y complejidad de las relaciones entre actores hace difícil definir una interdependencia clara entre factores. Creemos no obstante, que el número de usuarios y su preferencia por crear y compartir determinados tipos de contenidos, son una de las palancas de cambio que ejercen influencia a la hora de que los desarrolladores, distribuidores de software, instituciones y demás agentes implicados, apuesten por un desarrollo tecnológico que prime la eficiencia, la interoperabilidad, el acceso distribuido y la retro-compatibilidad.

Por todo ello, creemos que nuestra investigación contribuye a aportar mayor profusión y entendimiento de la importancia del usuario en el desarrollo tecnológico de la World Wide Web, y en especial del desarrollo del estándar HTML. En este sentido, también pensamos que se abre un nuevo horizonte para los Estudios Sociales de Ciencia y Tecnología, a la hora de considerar el papel del usuario y su influencia en este tipo de desarrollos tecnológicos.

\section{Bibliografía}

ANDERSON, Chris (2004): The Long Tail. Wired. http://www.wired.com/wired/archive/12.10/tail.html.

ANDERSON, Chris (2007) La Economía «Long Tail». De Los Mercados de Masas Al Triunfo de Lo Minoritario. Barcelona. Ediciones Urano.

ANDERSON, Paul (2007): What Is Web 2.0? Ideas, Technologies and Implications for Education http://www.jisc.ac.uk/media/documents/techwatch/tsw0701b.pdf.

ANDERSSON, David (2007): HTML5, XHTML2, and the Future of the Web. http://www. digital-web.com/articles/html5_xhtml2_and_the_future_of_the_web. 
BERNERS-LEE, Tim (2006): Reinventing HTML. http://dig.csail.mit.edu/breadcrumbs/ node/166.

BIJKER, Wiebe E. (1995): Sociohistorical Technology Studies. In Handbook of Science and Technology Studies, Revised Edition, edited by S. JASANOFF; G. MARKLE; J. Peterisen and T. PINCH, pp. 229-257. Thousand Oaks, CA: SAGE Publications. http://knowledge.sagepub.com/view/handbook-of-science-and-technology-studies/n11.xml.

BIJKER, Wiebe E.; T. P. HUGHES, and Trevor J. PINCH (1987): The Social Construction of Technological Systems: New Directions in the Sociology and History of Technology. Edited by Wiebe E. BIJKER; T. P. HUGHES and Trevor J. PINCH. Cambridge, MA: MIT Press.

BIJKER, Wiebe E. and Trevor J. PINCH (1984): The Social Construction of Facts and Artefacts: Or How the Sociology of Science and the Sociology of Technology Might Benefit Each Other. Social Studies of Science 14 (3): 399-441.

CALORE, Michael (2010): Scribd Ditches Flash for HTML5. Wired UK. http://www.wired.co.uk/news/archive/2010-05/07/scribd-ditches-flash-for-html5.

CASTELLS, Manuel (2001): La Galaxia Internet. Barcelona: Areté.

CASTRO, Elizabeth (2007): HTML, XHTML y CSS. Anaya Multimedia.

CHAU, M. L. C. (2009): HTML5: Its Evolution and Why It Matters. Itsc.org.sg: 9-20. http://www.itsc.org.sg/pdf/2011/Section2_Article1_021111.pdf.

DugGAN, Maeve and Aaron SMITH (2014): Three Technology Revolutions. Pew Research Internet Project. http://www.pewinternet.org/three-technology-revolutions/.

ECHEVERRÍA, Javier (2008): El Manual de Oslo y La Innovación Social. Arbor 184 (732): 609-618. http://arbor.revistas.csic.es/index.php/arbor/article/viewArticle/210.

ECHEVERRÍA, Javier (2013): Evaluar Las Innovaciones y su Difusión Social. Isegoría (48) (August 22): 173-184. doi:10.3989/isegoria.2013.048.09. http://isegoria. revistas.csic.es/index.php/isegoria/article/view/816/815.

ELLUL, Jacques (1964): The Technological Society. Vintage Books.

FRANGANILlo, J. (2010): Html5: El Nuevo Estándar Básico de La Web: 261-265. http://www.academia.edu/download/30940232/html5.pdf.

GONZÁlEZ García, Marta; José Antonio LÓPEZ CEREZO y José Luis LUJÁN (1996): Las Concepciones de La Tecnologia, en Ciencia, Tecnología y Sociedad. Una introducción al estudio social de la Ciencia y la Tecnología, 1-16. Madrid: TECNOS.

HACKETT, Edward J.; Olga AMSTERDAMSKA; Michael E. LYNCH and Judy WAJCMAN (2007): The Handbook of Science and Technology Studies. Edited by Edward J. HACKETT; Olga AMSTERDAMSKA; Michael E. LYNCH and Judy WAJCMAN. 3rd ed. Cambridge, MA: The MIT Press. 
HARDING, John (2010): YouTube API Blog: Flash and the HTML5 (video) Tag. http:// apiblog.youtube.com/2010/06/flash-and-html5-tag.html.

HELMER, Olaf; B. BROwn and T. GORDON (1966): Social Technology. New York: Basic Books.

HICKSON, lan and David HYATT (2008): HTML5 A Vocabulary and Associated APIS for HTML and XHTML (W3C Working Draft 22 January 2008). http://www.w3.org/ TR/2008/WD-html5-20080122/.

HUGHES, T. P. (1987): The Evolution of Large Technological Systems en The Social Construction of Technological Systems, edited by Wiebe E. BIJKER; T. P. HUGHES and Trevor J. PINCH, 51-82. Cambridge, MA: MIT Press.

INNERARITY, Daniel and Ander GURRUTXAGA (2009): ¿Cómo Es Una Sociedad Innovadora? Innobasque.

JOBS, Steve (2010): Thoughts on Flash. http://www.apple.com/hotnews/thoughtson-flash/.

KEITH, Jeremy (2010): HTML5 for Web Designers. Nueva York: A Book Apart.

KLEMPERER, Paul (2006): Network Effects and Switching Costs: Two Short Essays for the New Palgrave. SSRN Electronic Journal. doi:10.2139/ssrn.907502. http:// papers.ssrn.com/abstract $=907502$.

LANINGHAM, Scott (2006): DeveloperWorks Interviews: Tim Berners-Lee. August 22. http://www.ibm.com/developerworks/podcast/dwi/cm-int082206txt.html.

LE HEGARET, Philippe and lan JACOB (2009): Frequently Asked Questions (FAQ) about the Future of XHTML. http://www.w3.org/2009/06/xhtml-faq.html.

LIEBOWITZ, S. J. and Stephen E. MARGOLIS (1994): Network Externality: An Uncommon Tragedy. Journal of Economic Perspectives 8 (2). http://www.utdallas. edu/ liebowit/jep.html.

LINDER, Brad (2013): Cisco Sets H.264 Free(ish) with Royalty-Free Video Codec. Liliputing. http://liliputing.com/2013/10/cisco-sets-h-264-freeish-with-royaltyfree-video-codec.html.

LÓPEZ CEREZO, José A. and Marta I. GONZÁLEZ (2013): Encrucijadas Sociales de La Innovación. Isegoría (48) (August 22): 11-24. doi:10.3989/isegoria.2013.048.01. http://isegoria.revistas.csic.es/index.php/isegoria/article/view/808/807.

LUNDVALL, Bengt-Ake (1992): National Systems of Innovation: Towards a Theory of Innovation and Interactive Learning. London: Pinter Publ.

MULGAN, Geoff (2006): The Process of Social Innovation. Innovations: 145-162.

MULGAN, Geoff; Simon TUCKER; Rushanara ALI and Ben SANDERS (2007): Social Innovation. Edited by Skoll Centre for Social Entrepreneurship. The Basingstoke Press.

O'MARA, Mason P. (2012): New Features In HTML5. University of Wisconsin-Platteville (Department of Computer Science). 
O'REILLY, Tim (2005): What Is Web 2.0? Design Patterns and Business Models for the Next Generation of Software. O'Reilly. http://oreilly.com/web2/archive/what-isweb-20.html.

OECD (2005): Manual de Oslo: Guía Para La Recogida E Interpretación de Datos Sobre Innovación. OCDE Publishing

PILGRIM, Mark (2010): HTML5: Up and Running. O'Reilly Media. http://www.amazon. com/HTML5-Up-Running-Mark-Pilgrim/dp/0596806027.

QUINTANILLA, M. A.( 1998): Técnica y Cultura. Teorema XVII/3. http://www.oei.es/ salactsi/teorema03.htm.

QUINTANILLA, Miguel Ángel (1989): Tecnología: Un Enfoque Filosófico. Madrid: FUNDESCO.

ROGERS, Everett M. (1962): Diffusion of Innovations. New York. Free Press.

SCHAFER, Steven M. (2010): HTML, XHTML y CSS. Madrid: Anaya Multimedia.

SCHONFELD, Erick. (2010): Google, Mozilla, And Opera Take On H.264 With The WebM Project, A New Royalty-Free Video Codec. TechCrunch. http://techcrunch.com/2010/05/19/webm-google-h-264/.

SHAPIRO, Carl and Hal R. VARIAN (1999): Information Rules. Harvard Business School Press.

SLIDESHARE-BLOG (2011): SlideShare Moves to HTML5: Your Slides Will Work on All Mobile Devices Now. http://blog.slideshare.net/2011/09/27/slideshare-html5/.

THE MOZZILLA FOUNDATION \& OPERA SOFTWARE (2004): Position Paper for the W3C Workshop on Web Applications and Compound Documents. http://www. w3.org/2004/04/webapps-cdf-ws/papers/opera.html.

TUCKER, John V.; Victoria WANG and Kevin HAINES (2012): Phatic Technologies in Modern Society. Technology in Society 34 (1) (February): 84-93. doi:10.1016/j.techsoc.2012.01.001. http://linkinghub.elsevier.com/retrieve/pii/ S0160791X12000024.

VON Hippel, Eric (1998): The Sources of Innovation. Oxford: Oxford University Press.

VON Hippel, Eric (2005): Democratizing Innovation. Cambridge, MA: The MIT Press. http://web.mit.edu/evhippel/www/democ1.htm.

w3C (2011): HTML5. http://www.w3.org/TR/html5/.

W3C (2013): Differences from HTML4 (W3C Working Draft 28 May 2013). http://www. w3.org/TR/html5-diff/.

WANG, Victoria; John V. TUCKER; and Tracey E. RIHLL (2011): On Phatic Technologies for Creating and Maintaining Human Relationships. Technology in Society 33 (1-2) (February): 44-51. doi:10.1016/j.techsoc.2011.03.017. http://linkinghub. elsevier.com/retrieve/pii/S0160791X11000182.

WINNER, Langdon (1979): Tecnología Autónoma. Barcelona: Gustavo Gilli. 
WINNER (1980): Do Artifacts Have Politics ? Daedalus 109 (1): 121-136. https://blog. itu.dk/I-II-E2013/files/2013/11/winner-I-do-artifacts-have-politics.pdf.

WINNER (1993): Upon Opening the Black Box and Finding Empty: Social Constructivism and the Philosophy of Technology. Science, Technology \& Human Values 18 (3): 362-378. 